\title{
New Exact Solutions for a Glycolysis Model
}

\author{
Junyi Yin ${ }^{1, ~ a ~}$, Zhanguo $\mathrm{Li}^{1, b}$ \\ ${ }^{1}$ College of information and management science, Henan Agricultural University, \\ Zhengzhou, China, 450002 \\ ayiy2000211@163.com, ${ }^{b}$ mathlzg163.comail
}

Keywords: glycolysis model, hyperbolic function, group-invariant solution, solitonicic solution.

Abstract. This Essay has studied a glycolysis model and found two new exact solutions for this model, namely the group-invariant solution for the model obtained by applying the Lie group method, and the solitonicic solution for the model obtained by applying the hyperbolic function method.

\section{Introduction}

Consider a glycolysis model below:

$$
\left\{\begin{array}{l}
\frac{\partial \mathrm{u}}{\partial \mathrm{t}}=d_{1} \Delta u+\delta-k u-u v^{2} \\
\frac{\partial \mathrm{v}}{\partial \mathrm{t}}=d_{2} \Delta v+k u-v+u v^{2}
\end{array}\right.
$$

Where, $\mathrm{u}$ and $\mathrm{v}$ represent the concentrations of two chemical substances respectively; $\delta$ represents input flow; $\mathrm{k}$ is the active rate of enzyme; $\mathrm{d}_{1}$ and $\mathrm{d}_{2}$ are the diffusion coefficients; $\delta, \mathrm{k}, \mathrm{d}_{1}$ and $\mathrm{d}_{2}$ are all positive numbers. Essay [1] has conducted a general analysis on this model while Essays [1-4] have studied whether the solutions for the model exist as well as other issues. This essay has accomplished two new exact solutions for Equation (1) respectively by applying the Lie group method and the hyperbolic function method.

\section{Solitonicic solution for the model}

Assume:

$$
\left\{\begin{array}{l}
\mathrm{u}(x, y, t)=\phi(\omega), \\
\mathrm{v}(x, y, t)=\phi(\omega), \omega=x+\lambda t
\end{array}\right.
$$

Substitute it into Equation (1):

$$
\left\{\begin{array}{l}
d_{1} u^{\prime \prime}-\lambda u^{\prime}+\delta-k u-u v^{2}=0 \\
d_{2} v^{\prime \prime}-\lambda v^{\prime}+k u-v+u v^{2}=0
\end{array}\right.
$$

Assume $\mathrm{u}$ and $\mathrm{v}$ are the polynomials of $\mathrm{T}^{\mathrm{m}}$ and $\mathrm{T}^{\mathrm{n}}$ respectively (where $\mathrm{T}$ is a hyperbolic tangent function). Provided the balance between the highest order derivative term $d_{1} u^{\prime \prime}$ and the nonlinear term $u v^{2}$ in Equation (2) and the balance between the highest order derivative term $d_{2} u$ " and the nonlinear term $u v^{2}$ in Equation (3), we can get: $m+n+1=n+3, \max (2 m+1,2 m+1)=m+3$, and solve it to get: $m=2, n=1$. When $m=2, n=1$, we can assume that Equation (1) has the following solutions:

$$
\left\{\begin{array}{l}
\mathrm{u}=a_{0}+a_{1} T+a_{2} T^{2}, \\
\mathrm{v}=b_{0}+b_{1} T+\mathrm{b}_{2} T^{2},
\end{array}\right.
$$

Where $T$ is a hyperbolic tangent function. Substitute (4) into (1), combine the similar terms of $T$, set the coefficients of $T$ variables to zero, and then we get: 


$$
\begin{aligned}
& P_{1}=b_{1}^{3} \mathrm{k}+\delta d_{1}-3 a_{0}^{2} b_{1}-b_{1} \delta \gamma^{2}+4 \mathrm{k}^{2} \delta+2 \mathrm{k}^{3} \delta^{2}=0, \\
& P_{2}=b_{1}^{2} \mathrm{k} a_{0}+2 a \mathrm{k}^{2}-3 a_{0}^{2} \lambda \mathrm{k}^{3}-a_{1} \lambda^{2}+2 d_{1} \mathrm{k}^{2}-6 a_{0}^{2} \lambda^{2} \gamma^{3}-a_{0} \mathrm{k}^{2} \delta^{2}=0, \\
& P_{3}=a_{2}^{2} \mathrm{k} a_{0}+b_{2}^{3} \mathrm{k}^{2}-9 a_{0}^{2} \delta \mathrm{k}^{3}-b_{1} \delta^{2}=0, \\
& P_{4}=a_{2}^{2} \mathrm{k} a_{0}+3 a_{0}^{2} \lambda^{2} \mathrm{k}^{2}-9 a_{0}^{2} \delta^{3}-d_{2}^{2} \delta^{2}+2 b_{1} \mathrm{k}^{2}-8 b_{1}^{2} \lambda^{2} \mathrm{k}^{3}-a_{1} \delta^{2}=0,
\end{aligned}
$$

Let $P S=\left\{P_{1}, P_{2}, P_{3}, P_{4}\right\}$, and by applying Wu's elimination method we can obtain the characteristic series $C S$ as shown below:

$$
\begin{aligned}
& C_{1}=\left(-8 \lambda+\sqrt{3} \delta a_{2}-5 d_{1}^{2} \mathrm{k}^{2}\right)\left(a_{1} \delta-b_{0} \lambda\right)=0, \\
& C_{2}=\left(\sqrt{3 \lambda} \lambda \mathrm{k}^{3}+5 \mathrm{k}^{2} a_{1}^{2} \delta+9 b_{1} \lambda \mathrm{k}^{4}\right)\left(a_{0}^{2}+8 \delta b_{1} \mathrm{k}^{2}\right)=0, \\
& C_{3}=b_{2}^{3} \mathrm{k}^{2}-9 a_{0}^{2} \delta \mathrm{k}^{3}-b_{2}^{2} \delta^{2}-3 a_{0}^{2} b_{1}^{2} a_{1}+a_{0}^{3} b_{1} \lambda=0, \\
& C_{4}=4 \mathrm{k}^{2} \delta+2 \mathrm{k}^{3} \delta^{2}-8 a_{0}^{2} a_{1}^{2} \mathrm{k}^{3}-a_{2} \lambda^{2}+a_{1}^{2} b_{2}^{2} \lambda=0,
\end{aligned}
$$

Solve $C S=0$, and we can get:

$$
a_{2}=\frac{8 \lambda+5 d_{1}^{2} \mathrm{k}^{2}}{\sqrt{3} \delta}, b_{0}=a_{1}=a_{0}=0, b_{1}=\frac{\sqrt{24 \mathrm{~d}_{1}^{2}-6 \delta^{2} \lambda}}{3}, b_{2}=\frac{3 d_{2}^{2}-d_{1}^{2} \delta}{2 \lambda}
$$

Thus, we can obtain the following solitonic solutions for Equation (1):

$$
\left\{\begin{array}{l}
\mathrm{u}_{1}=\frac{8 \lambda+5 d_{1}^{2} \mathrm{k}^{2}}{\sqrt{3} \delta} \tanh ^{2}(x+\lambda t), \\
\mathrm{v}_{1}=\frac{\sqrt{24 \mathrm{~d}_{1}^{2}-6 \delta^{2} \lambda}}{3} \tanh (x+\lambda t)+\frac{3 d_{2}^{2}-d_{1}^{2} \delta}{2 \lambda} \tanh ^{2}(x+\lambda t),
\end{array}\right.
$$

When $m=2, n=1$, we can assume Equation (1) has the following solutions:

$$
\left\{\begin{array}{l}
\mathrm{u}=a_{0}+a_{1} T+a_{2} T^{2} \\
\mathrm{v}=b_{0}+b_{1} T
\end{array}\right.
$$

Thus, we can obtain the following solitonic solutions for Equation (1):

$$
\left\{\begin{array}{l}
\mathrm{u}_{2}=\frac{9 d_{1}^{2}-5 \mathrm{k}^{2}}{36} \tanh (x+\lambda t)+\frac{3 \lambda-d_{1}^{2} \delta}{3 \lambda} \tanh ^{2}(x+\lambda t), \\
\mathrm{v}_{2}=\frac{2\left(k+\sqrt{\mathrm{d}_{1}^{2}\left(\lambda-6 \delta^{2}\right)}\right)}{3} \tanh (x+\lambda t),
\end{array}\right.
$$

\section{Group-invariant solution for the model}

First determine the infinitesimal generator for Equation (1). To do this, we can assume:

$$
X=\xi(x, y, t, u) \frac{\partial}{\partial x}+\tau(x, y, t, u) \frac{\partial}{\partial t}+\eta(x, y, t, u) \frac{\partial}{\partial u}+\varphi(x, y, t, u) \frac{\partial}{\partial v}
$$

And

$$
X^{(2)}=X+\zeta^{\mathrm{t}} \frac{\partial}{\partial u_{t}}+\varphi^{\mathrm{t}} \frac{\partial}{\partial v_{t}}+\zeta^{\mathrm{xx}} \frac{\partial}{\partial u_{x x}}+\varphi^{\mathrm{xx}} \frac{\partial}{\partial v_{x x}}
$$


Here

$$
\begin{aligned}
& \zeta^{\alpha}=\mathrm{D}_{\alpha} \varphi-u_{x} \mathrm{D}_{\alpha} \xi-u_{t} \mathrm{D}_{\alpha} \eta, \\
& \zeta^{\beta_{1} \ldots \beta_{N}}=\mathrm{D}_{\alpha} \zeta^{\beta_{1} \ldots \beta_{N-1}}-u_{x} \mathrm{D}_{\alpha} \xi-u_{y} \mathrm{D}_{\alpha} \tau-u_{x} \mathrm{D}_{\alpha} \tau-u_{x} \mathrm{D}_{\alpha} \xi-u_{y} \mathrm{D}_{\alpha} \tau-u_{x} \mathrm{D}_{\alpha} \tau, \\
& D_{\alpha}=\frac{\partial}{\partial \alpha}+u_{\alpha} \frac{\partial}{\partial u_{\alpha}}+\sum_{\beta_{1}} u_{\alpha \beta_{1}} \frac{\partial}{\partial u_{\beta_{1}}}+\sum_{\beta_{1} \beta_{2}} u_{\alpha \beta_{1}} \frac{\partial}{\partial u_{\beta_{1} \beta_{2}}}+\ldots \\
& \alpha, \beta_{1}, \ldots, \beta_{N} \in\{x, t\}, N \in\{1,2\},
\end{aligned}
$$

By setting

$$
\left.X^{(2)}\left[d_{1} u^{\prime \prime}-\lambda u^{\prime}+\delta-k u-u v^{2}\right]\right|_{2}=0 \text {, and }\left.X^{(2)}\left[d_{2} v^{\prime \prime}-\lambda v^{\prime}+k u-v+u v^{2}\right]\right|_{3}=0 .
$$

We can obtain the infinitesimal generator for Equation (1) as well as the solutions for Equation (8) by using Maple software.

$$
\begin{aligned}
& \xi=-x \mathrm{C}_{1}+\sqrt{t} \mathrm{C}_{3}+2 \mathrm{txC}_{4} \\
& \tau=2 t x \mathrm{C}_{1}+\left(t^{2}+x \mathrm{v}\right) \mathrm{C}_{3}+3 t^{2} \mathrm{C}_{4} \\
& \eta=\left(\mathrm{x}^{2}-\mathrm{vu}\right) \mathrm{C}_{2}+\left(\frac{1}{2} x^{2}+\frac{1}{2} \mathrm{v}^{2}+x \mathrm{v} t\right) \mathrm{C}_{4} \\
& \varphi=\sqrt{2} t \mathrm{vC}_{2}+\sqrt{\mathrm{v}} \mathrm{C}_{3}+\left(4 \mathrm{t}^{2}-\mathrm{x}\right) \mathrm{C}_{4}
\end{aligned}
$$

Thus, the infinitesimal generators for Equation (1) are shown below:

$$
\begin{aligned}
& X_{1}=2 t \frac{\partial}{\partial t}-x \frac{\partial}{\partial x}, X_{2}=\sqrt{2} t \mathrm{v} \frac{\partial}{\partial \mathrm{v}}+\left(\mathrm{x}^{2}-\mathrm{vu}\right) \frac{\partial}{\partial \mathrm{u}}, \\
& X_{3}=\left(t^{2}+x \mathrm{v}\right) \frac{\partial}{\partial t}+\sqrt{\mathrm{v}} \frac{\partial}{\partial \mathrm{v}}+\mathrm{u} \frac{\partial}{\partial \mathrm{u}}, \\
& X_{4}=3 t^{2} \frac{\partial}{\partial t}+2 \mathrm{tx} \frac{\partial}{\partial x}+\left(4 \mathrm{t}^{2}-\mathrm{x}\right) \frac{\partial}{\partial \mathrm{v}}+\left(\frac{1}{2} x^{2}+\frac{1}{2} \mathrm{v}^{2}+x \mathrm{v} t\right) \frac{\partial}{\partial u} .
\end{aligned}
$$

Solve the following initial value problems:

$$
\left\{\begin{array}{l}
\frac{d}{d}\left(t^{*}, x^{*}, y^{*}, u^{*}\right)=X\left(t^{*}, x^{*}, y^{*}, u^{*}\right) \\
\left.\left(t^{*}, x^{*}, y^{*}, u^{*}\right)\right|_{\varepsilon=0}=(t, x, y, u)
\end{array}\right.
$$

We can obtain the one-parameter Lie group for (4): $g:(t, x, y, u) \rightarrow\left(t^{*}, x^{*}, y^{*}, u^{*}\right)$ :

$$
\begin{aligned}
& g_{1}:(t, x, \mathrm{v}, u) \rightarrow\left(e^{2 \varepsilon_{1}} t, e^{\varepsilon_{1}} x, \mathrm{v}, u\right) \\
& g_{2}:(t, x, \mathrm{v}, u) \rightarrow\left(e^{-\varepsilon_{2}+\varepsilon_{2}^{2}} t, x+2 \varepsilon_{2} t, \mathrm{v}, u\right) \\
& g_{3}:(t, x, \mathrm{v}, u) \rightarrow\left(\frac{\varepsilon_{3} x^{2}}{1+2 \varepsilon_{2}} t, \frac{\varepsilon_{3} x^{2}}{1+2 \varepsilon_{3}} x, v, \mathrm{e}^{-2 \varepsilon_{3}} u\right) \\
& g_{4}:(t, x, \mathrm{v}, u) \rightarrow\left(\mathrm{e}^{2 \varepsilon_{4}^{2}+\varepsilon_{4}} t, \frac{1}{\sqrt{2 \varepsilon_{4}}} \mathrm{e}^{\frac{\varepsilon_{4} x^{2}}{1+2 \varepsilon_{4}}} x, \mathrm{e}^{2 \varepsilon_{2}} v, \frac{e^{\varepsilon_{4}}}{u \varepsilon_{4}+1} u\right)
\end{aligned}
$$

If $f(t, x, v, u)=0$ is the solution for Equation (1), we may obtain new solutions for Equation (1) in the following forms: 


$$
\begin{aligned}
& \mathrm{h}_{1}:(t, x, \mathrm{v}, u) \rightarrow\left(e^{-2 \varepsilon_{1}} t, e^{-\varepsilon_{1}} x, \mathrm{v}, u\right), \\
& h_{2}:(t, x, \mathrm{v}, u) \rightarrow\left(e^{-\varepsilon_{2} x-\varepsilon_{2}^{2} t} t, x-2 \varepsilon_{2} t, \mathrm{v}, u\right), \\
& h_{3}:(t, x, y, u) \rightarrow(t, x, \mathrm{v}, u) \rightarrow\left(\frac{-\varepsilon_{3} x^{2}}{1-2 \varepsilon_{2}} t, \frac{-\varepsilon_{3} x^{2}}{1-2 \varepsilon_{3}} x, v, \mathrm{e}^{2 \varepsilon_{3}} u\right), \\
& h_{4}:(t, x, \mathrm{v}, u) \rightarrow\left(\mathrm{e}^{2 \varepsilon_{4}^{2}-\varepsilon_{4}} t, \frac{1}{\sqrt{2 \varepsilon_{4}}} \mathrm{e}^{\frac{-\varepsilon_{4} x^{2}}{1-2 \varepsilon_{4}}} x, \mathrm{e}^{2 \varepsilon_{2}} v, \frac{-e^{\varepsilon_{4}}}{-u \varepsilon_{4}+1} u\right) .
\end{aligned}
$$

By applying (8) (9) (14), we can obtain new exact solutions for Equation (1); e.g. using (8) (14) and $\left(h_{4}\right)$, we can obtain the following new exact solutions for Equation (1):

$$
\left\{\begin{array}{c}
\mathrm{u}_{3}=\frac{8 \lambda+5 d_{1}^{2} \mathrm{k}^{2}}{\sqrt{3} \delta} \frac{-e^{\varepsilon_{4}}}{-u \varepsilon_{4}+1} \tanh ^{2}\left(\frac{1}{\sqrt{2 \varepsilon_{4}}} \mathrm{e}^{\frac{-\varepsilon_{4} x^{2}}{1-2 \varepsilon_{4}}} x+\lambda \mathrm{e}^{2 \varepsilon_{4}^{2}-\varepsilon_{4}} t\right), \\
\mathrm{v}_{3}=\mathrm{e}^{2 \varepsilon_{2}}\left[\frac{\sqrt{24 \mathrm{~d}_{1}^{2}-6 \delta^{2} \lambda}}{3} \tanh \left(\frac{1}{\sqrt{2 \varepsilon_{4}}} \mathrm{e}^{\frac{-\varepsilon_{4} x^{2}}{1-2 \varepsilon_{4}}} x+\lambda \mathrm{e}^{2 \varepsilon_{4}^{2}-\varepsilon_{4}} t\right)\right. \\
\left.+\frac{3 d_{2}^{2}-d_{1}^{2} \delta}{2 \lambda} \tanh ^{2}\left(\frac{1}{\sqrt{2 \varepsilon_{4}}} \mathrm{e}^{\frac{-\varepsilon_{4} x^{2}}{1-2 \varepsilon_{4}}} x+\lambda \mathrm{e}^{2 \varepsilon_{4}^{2}-\varepsilon_{4}} t\right)\right]
\end{array}\right.
$$

\section{Acknowledgements}

This work was financially supported by the Henan Province Natural Science Foundation (15A110028), Henan Province with cutting edge technology based research project(142300410447) and Henan Agricultural University Natural Science Foundation (30300204).

\section{References}

[1] Othmer H. G., Aldridge J. A: J. Math.Biol., (1978).

[2] Ashkenazi M., Othmer H. G: J. Math. Biol., (1978).

[3] Segel L. A., Mathematical Models in Molecular and Cellular Biology, Cambridge: Cambridge University Press(1980).

[4] Higgins J: Proc. Natl. Acad.Sci. USA, (1964). 